\title{
Concept of a Robot for Interior Building Trades by the Example of Wall Slits in Masonry
}

\author{
Dieter Spath, Jürgen Andres \\ wbk - Institute for Machine Tools and Production Science, \\ University of Karlsruhe (TH), Kaiserstr. 12, D-76128 Karlsruhe, Germany
}

\begin{abstract}
This article deals with the automation of interior building trades by the use of robots. Therefore, the specifications of a robot system for the manufacturing of wall slits, as a preliminary step of wiring and electrical installations in masonry construction, will be defined. The main motivation to reduce the manual tasks is the great difference between the high qualification of workers (especially electricians) and partly the unpretending hard work which does not require such a professional education in the same way. So, the focus of the paper lays on a manufacturing process that is suitable for automation, kinematical concepts for the robot and the development of an appropriate end effector. The requirements of the closeness of space and light weight result in the necessity of other kinematical concepts and structures, because common structures of industrial robots are not suitable. The presented kinematics are based on linear components as best solution to cope with the two-dimensional area and a special developed wrist to bridge the existing clearance volumes of the main axes and to allow the accessibility of edges. Furthermore, the robot has to be able to reach working heights near to ceilings and simultaneously to pass a door. Besides this, a robotized process will be explained in order to reduce manual refinishing operations as much as possible because of economical reasons. Consequently, the design of an appropriate end effector based on a joint cutter will be shown. Finally, demands for the programming system and further employments of the robot will be given as a short preview.
\end{abstract}

\section{$1 \quad$ Motivation}

The situation in the construction industry in Europe and especially in Germany is impressed by an increasing lack of skilled workers and simultaneously by an increasing compulsion for improving productivity and economic efficiency. In Germany the construction of residential buildings is estimated about $50 \%$ of the whole construction business. After residential buildings are finished in the rough, 
particularly block and brick work, many tasks prove to be on a low level of productivity as well. The degree of mechanization amounts to $8 \%$ only $/ 1,2 /$.

For example, the job description of an electrician is characterized by the difference of a high skillness and often unpretending and strenuous work, like the insertion of wall slits in masonry. Informations of the electric associations are that about the half of the time to complete electrical installations is needed for wall slits. Not at last, the lack of new production technologies also leads to a wandering of expert workers to other, more attractive businesses. The technical frankness of electricians and their know how of control technologies above average allow a good forecast of the introduction of robotics in this field of construction.

\section{Kinematical specifications for an Indoor Robot}

The zones of electrical installations in masonry represent the main requirements (see fig. 3) for the conceptuation of the appropriate kinematical structure. Approaches to use common kinematics of industrial robots with six rotational axes do not fit because of several reasons. Firstly, the useful range for operations in and at walls is only about $12 \%$ of the whole range. Secondly, robots with such a configuration and a prospected reach up to ceilings have an enormous weight that exceed the common stress on floors about $425 \mathrm{~kg} / \mathrm{m}^{2}$. At last, walls slits are only necessary in horizontal or vertical dimension. The requirements of installation zones demand a main axes' structure similar to a plotter.

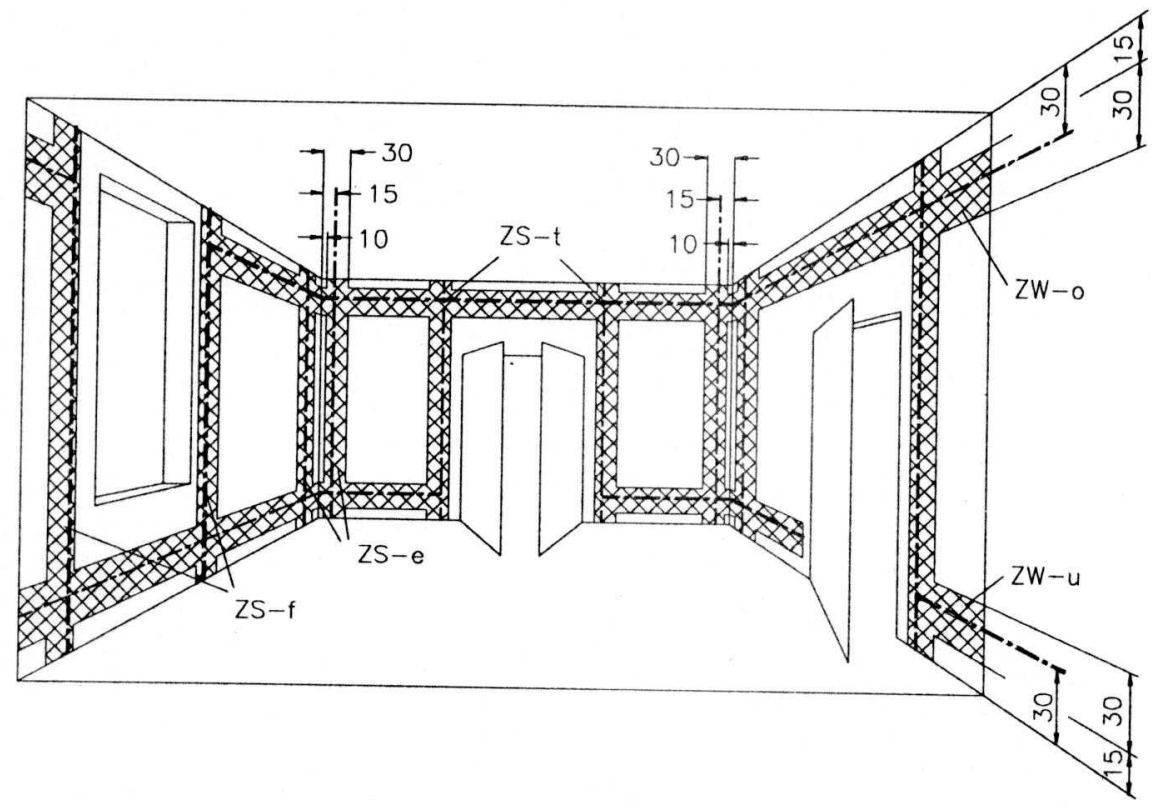

fig. 1: $\quad$ areas of electrical installations /3/ 
Other restrictions derive from two necessities. On one hand the robot has to reach heights up to the ceiling and on the other hand it has to be able to pass doors independent of the autonomy of the system. So, a telescopic, vertical axis is needed to fullfill these antithetical demands.

In order to cover a great range of walls to be processed a market study done in the European project ROCCO serves as a basis for the further design of the robot system. So, the already existing prototype of a mobile masonry robot and the post processing robot constitute an integral system for masonry tasks. Consequently, it is possible to process about $80 \%$ of occuring walls with a vertical extension of $3.5 \mathrm{~m} / 2 /$.

Further kinematical demands result from the necessity to reach corners of rooms very closely. Because of existing clearance volumes by linear axis a special developed wrist to bridge these volumes is necessary. Analogous to other developments in the field of service robots a real integration of kinematical features and drive technologies has to be carried out. This means a usage of the structure of drives as a kinematical element to a great possible extent at the same time. This circumstance leads to the reduction of weight and also to an economic realisation of the system.

A further requirement is modularity because the whole system has to be able to be taken apart easily. Mainly, this is needed for transportation reasons on the construction site. Another intention is the exchangeability of components to adapt the robot system to environmental changes or other possible indoor operations.

In contrary to common factory environments other environmental conditions prevail on construction sites caused by varying temperatures, dust by parallel construction operations and moist. These circumstances result in a high robustness in the mechanical structure, especially for bearings, guideways and electrical components.

\section{Process Structuring and End Effector}

To structure the process for an automated operation and the design of an appropriate end effector basic examinations on the manual process and several standards are carried out. Lengths and depths of wall slits are dependent on the arrangement of switches, sockets, junction boxes and electrical distributors as well as the kind of electrical wirings like for example lights, electrical cookers and broadband cables $/ 3,4,5 /$.

Common tools for the manual manufacturing of wall slits rule out for the employment of robots. The impact of drilling hammers will be to high for the robot system and high qualities are not possible. Milling tools are able to reduce manual refinishings but the cutting forces are to high. Different to these tools commonly used joint cutters have the smallest process forces but the arrangement of 
the grinding wheel leads to considerable manual refinishings. Therefore, the advantages of each process have to be combined. The follwing tabular shows the feasible feeds of the two possible processes with identical cutting power for different cross-sections of wall slits.

\begin{tabular}{|l|c|c|c|c|c|}
\hline $\begin{array}{l}\text { number of hollow tubes } \\
\text { diameter [mm] }\end{array}$ & $1 * \varnothing 12.0$ & $2 * \varnothing 12.0$ & $3 * \varnothing 12.0$ & $4^{*} \varnothing 12.0$ & $1^{*} \varnothing 22.5$ \\
\hline feed $[\mathrm{mm} / \mathrm{s}]$ for milling & 2.22 & 1.11 & 0.74 & 0.55 & 0.59 \\
\hline feed $[\mathrm{mm} / \mathrm{s}]$ for grinding & 3.91 & 2.76 & 2.46 & 2.14 & 2.08 \\
\hline
\end{tabular}

Actually, the developed end effctor is based on a joint cutter, but the grinding wheel is inclined $45^{\circ}$. This leads to a temporally shifted processing of the two sides of the slits. At first, the grinding wheel dives under $45^{\circ}$ nominal pressure angle into the wall. The next steps are the cutting of the horizontal vertical line, disengaging the effector, turning $180^{\circ}$, renewed engaging of the tool and retracing the same line. The outcome is a v-shaped wall slit that does not need any manual refinishings. A following programming of geometric and technological data has to take into consideration the offset of the tool center point.
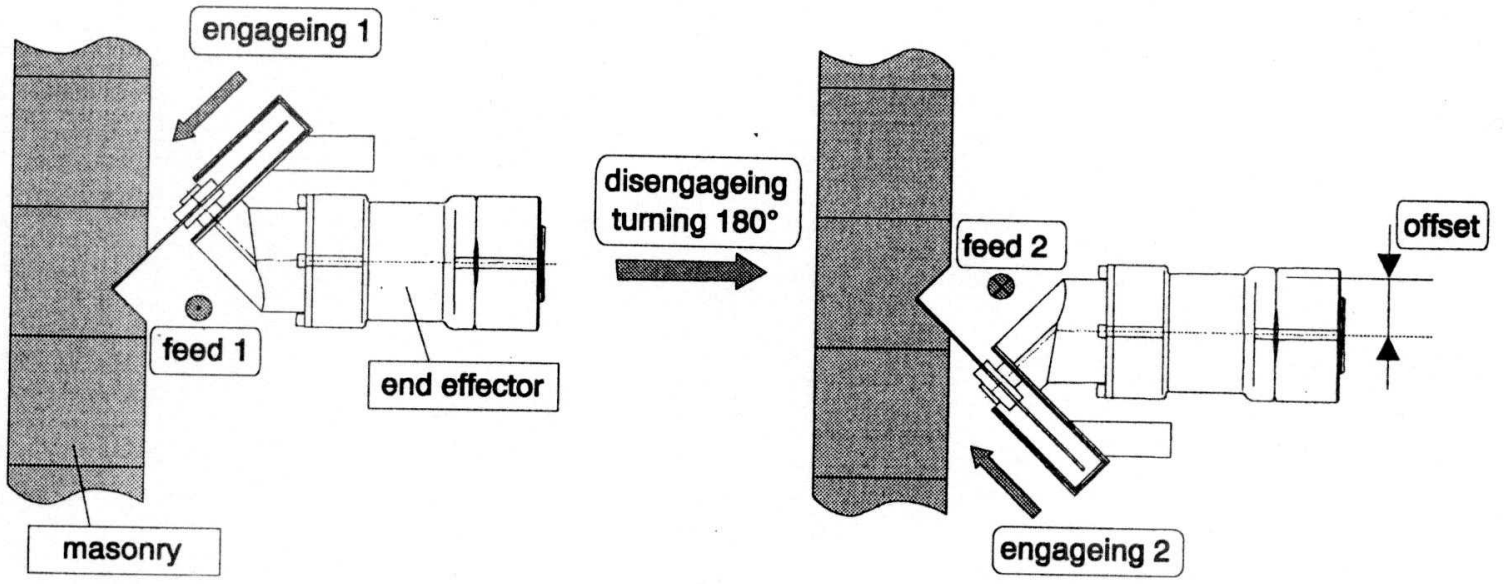

fig. 2: $\quad$ operation of the end effector and offset

In order to enable the processing of corners another manufacturing strategy is obligatory because an engageing under $45^{\circ}$ is not possible. Therefore, the grinding wheel dives into the wall vertically. This operation has not been possible with conventional tools for grinding or milling up to now. Here, two parallel cuttings are done and the segment has to be breaked out manually. This manual operation will be an acceptable effort since these kinds of wall slits occur rarely. At the initial stage the tool can be also used to produce gaps for electrical power points to avoid tool changes. 


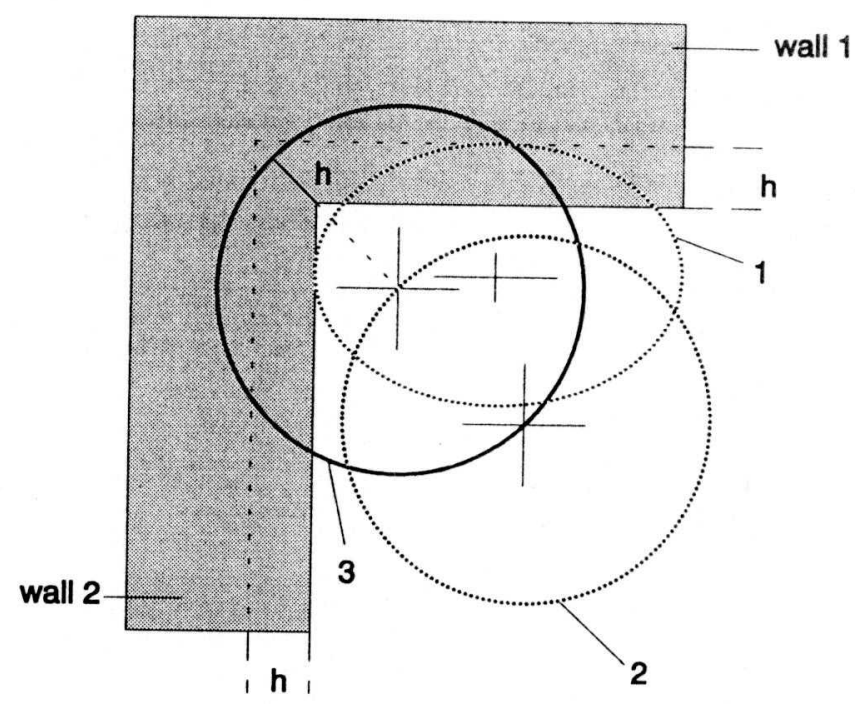

fig. 3: $\quad$ processing of a corner

\section{Conceptual Design of the Indoor Robot}

The following described concept for the indoor robot does not consider the necessary mobile plattform that influences particularly the autonomy of the robot system. Rather the kinematical design of the main axes and the wrist is examined.

The main axes' kinematics consists of a telescopic, vertical axis similar to a fork lift reach truck. This allows passing doors and operating at ceiling heights. The mechanism of the telescopic arm does not only serve for the processing but also for a tight fixture of the whole robot system between the floor and the ceiling. Consequently, the forces and moments on the system can be compensated during the process and also the tilt resistance is guaranteed.

The actuation is done by compact hollow shaft drives and a toothed belt or a ball screw. An integrated overload release stops the extension of the telescope. Because of different operation heights the mechanism of actuation of the actual positioning range has to allow alternations of length. Therefore a second belt with appropriate defelection pulleys is provided. So, the main axis' movement is independent from the vertical adjustment and clamping.

For horizontal movements a common linear axis is intended. Because of reasons for adaption to environmental conditions and other applications the connection between the two main axis shall realized as a plug-in module for centred fixture and power supply. This modularity qualifies for an easy transportation and assembly of the system and also for the use of different horizontal axes for narrow alcoves. Figure 4 shows the mechanical structure of the main axes. 


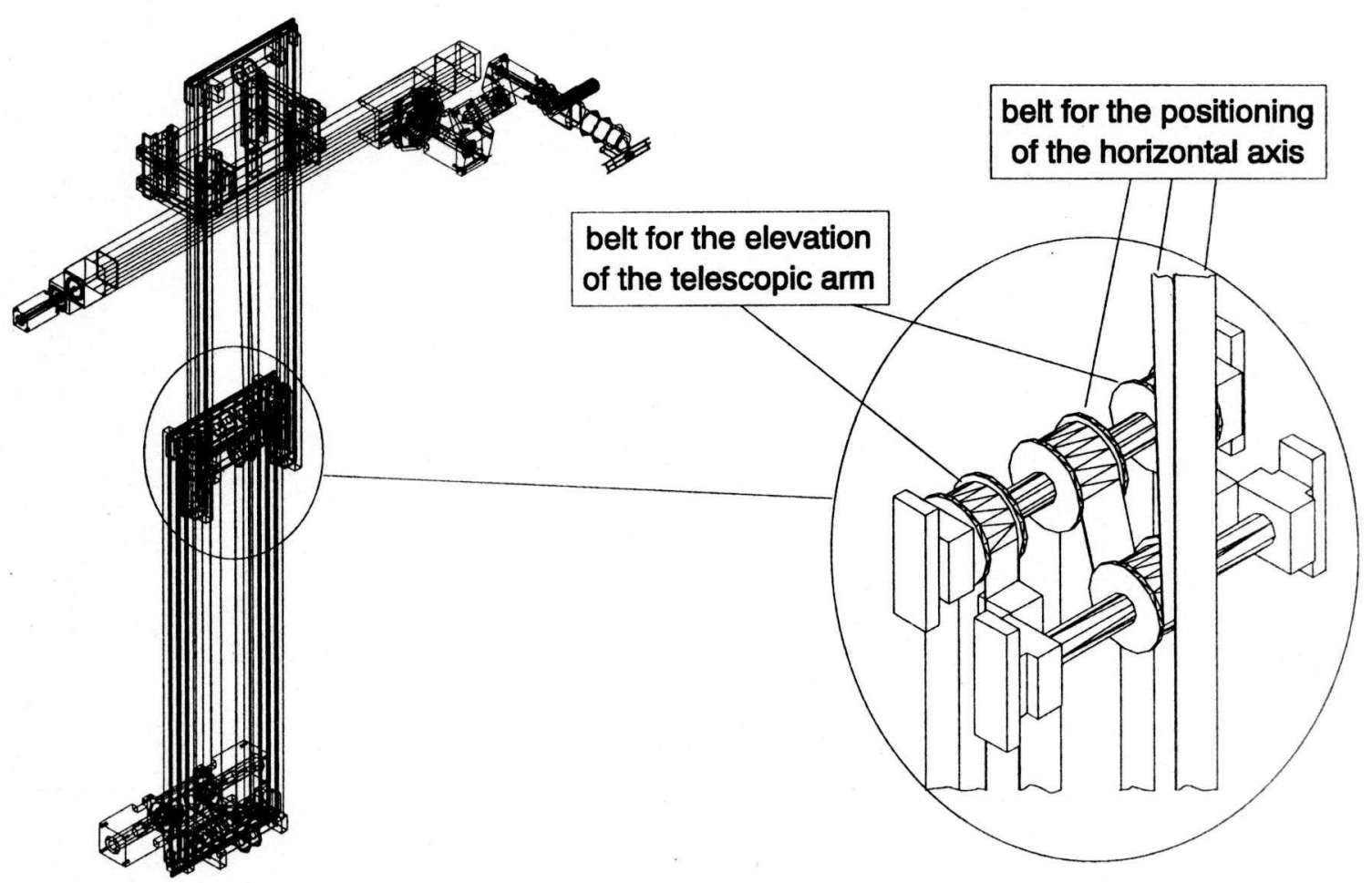

fig. 4: $\quad$ mechanical structure of the indoor robot without mobile platform and deflection pulleys

The slide unit to carry the horizontal axis is provided with two parallel roller units. This enables an engageing in both the upper and lower part of the telescope's guideways. The design of the slide must be tightly set to prevent inaccuracies through tiltings and to allow a continuous transition between the two parts of the extension axes.

To design the robot's wrist several requirements have to be taken into account. Firstly, the clearance volumes through the linear units and the planned mobile platform have to be bridged. However, common wrists of robots are not suitable since they do not satisfy the demands of light weight and small dimensions. Wrists based on a differential movements are not applicable because they cannot compensate the clearance volumes and retain the occuring stresses. On the opposite, the employment of hollow shaft drives serves for a sufficient summening up of weights and process forces as well as for a compact structure of the wrist. Their usability as a kinematical component brings about additional advantages like a light, rather reasonable construction and a high integration of actuators and arm links.

Figure 5 shows the mechanical structure of the wrist with three actuators that are tilted $45^{\circ}$ against the horizontal. These kinematics fulfill the above mentioned requirements and allow also the so called 'grip around the corner'. Consequently, 
it is possible to operate at the lateral wall, too. By that, all cases of necessary operations can be covered with simultaneously small collision volumes by respective tiltings of the actuators of $90^{\circ}$ (i.e. the cutting of parallel and v-shaped walls slits in all installation areas and corners). If the componentized construction has to be implemented as a plug-in module as well this will lead to the same advantages already discussed for the main axes.

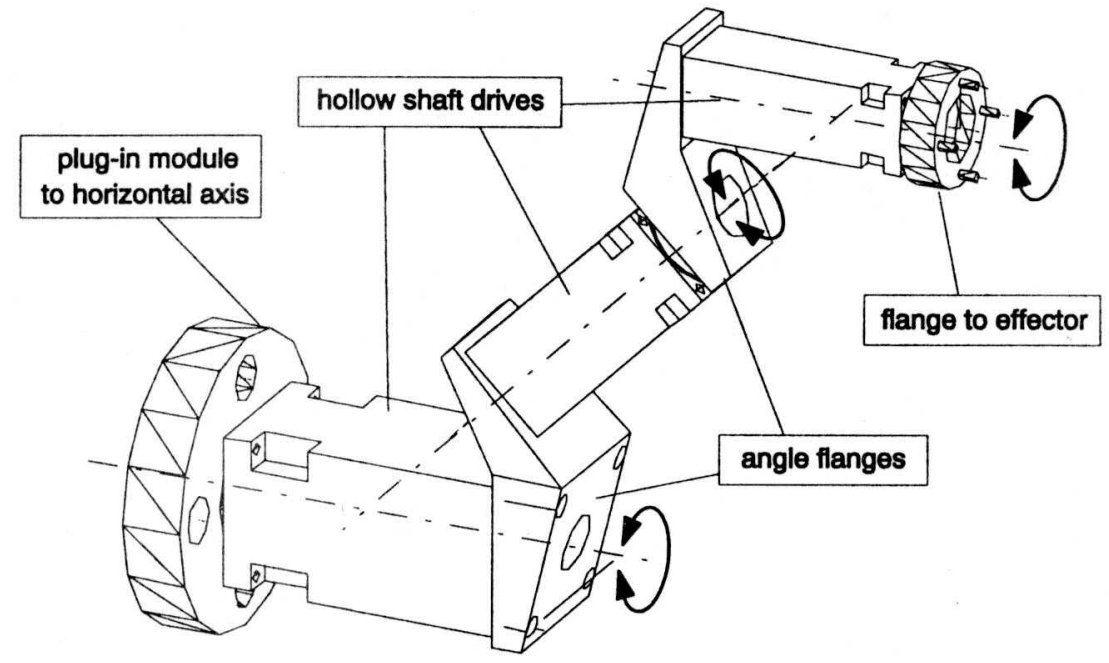

fig. 5: $\quad$ concept of the wrist

The engageing of the end effector is not done by an overlapped movement of the main axes but by a linear actuator. The actuator is directly connected with the end effector and the movement is parallel to the diameter of the grinding wheel. Consequently, this independence avoids more complex calculations for the path planning caused by the different shapes of wall slits.

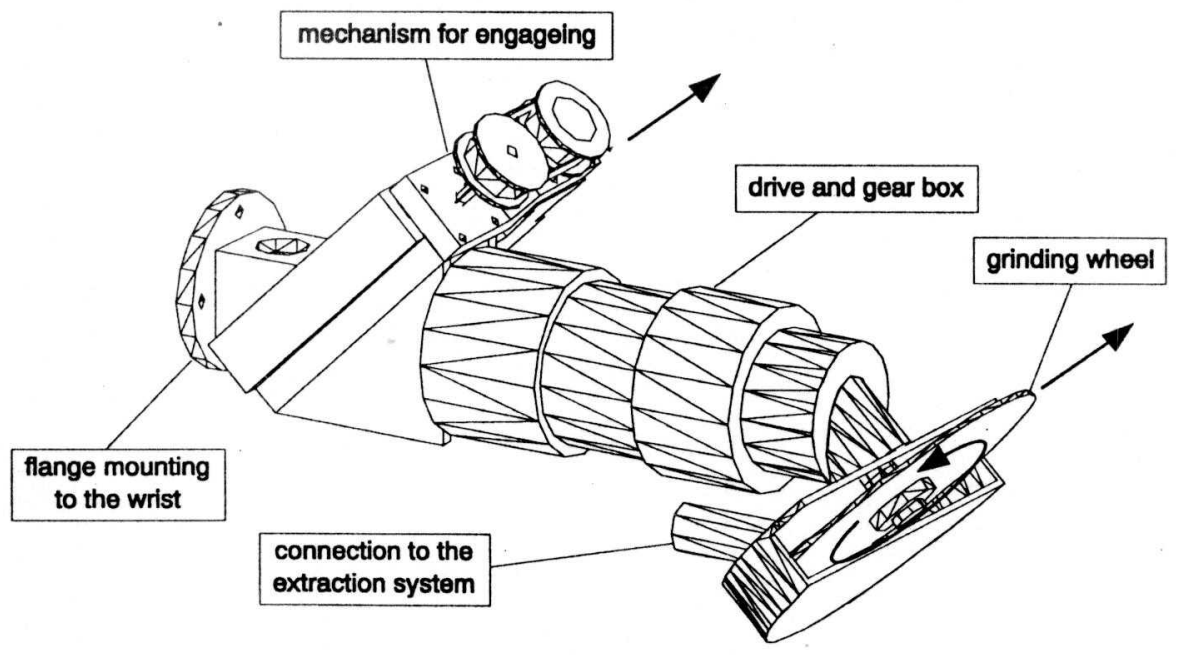

fig. 6: $\quad$ end effector with device for engageing 
This article describes the kinematical concept of a robot for interior building trades and a specific process automation for the cutting of wall slits in masonry. At the moment the automation process is implemented in a test bed at the institute's robot laboratory by using a prototype of the end effector. These examinations serve for the development of an appropriate programming system with functional macros. At the initial stage, the programming system is not implemented with a direct CAD-connection but with a user-friendly graphical interface for a fast realisation and a higher acceptance on the site. However, the system has to be open also for highly integrated construction processes in the future.

Some interests are also lying in the spreading into other interior building trades (e.g. tiling, grouting and painting) and into the field of pre-fabrication. Because of the system's modularity it can be used for these tasks by reasonable adaptions or even exchangability of components.

\section{References}

/1/ Spath, D.; Andres, J.; Bock, T.; Steffani, H.F.: Flexible Automatisierung im Mauerwerksbau, in: Autonome Mobile Systeme 1994, Springer, Berlin Heidelberg, 1994

/2/ Andres, J.; Bock, T.; Gebhart, F.; Steck, W.: First Results of the Development of the Masonry Robot System ROCCO: a Fault Tolerant Assembly Tool, in: Proceedings of the 11th ISARC, Elsevier, Amsterdam, 1994

/3/ DIN 18015 Teil 3: Elektrische Anlagen in Wohngebäuden Leitungsführung und Anordnung von Betriebsmitteln, Beuth, Berlin, 1990

/4/ FTZ 731 TR 1: Rohrnetze und andere verdeckte Führungen für Fernmeldeleitungen in Gebäuden, Deutsche Telekom, 1993

/5/ Thierholf, H.: Aussparungen und Schlitze im Mauerwerk zulässig, in: der elektromeister + deutsches elektrohandwerk 2/1990, pages 74-76 